\title{
On the total weight of weighted matchings of segment graphs
}

\author{
Thomas Stoll \\ School of Computer Science \\ University of Waterloo, Waterloo, ON, Canada N2L3G1 \\ tstoll@cs. uwaterloo.ca \\ Jiang Zeng \\ Institute Camille Jordan \\ Université Claude-Bernard (Lyon I) \\ 69622 Villeurbanne, France \\ zeng@math.univ-lyon1.fr
}

Submitted: Apr 30, 2008; Accepted: Apr 24, 2009; Published: Apr 30, 2009

Mathematics Subject Classification: 11D45, 05A15, 33C45

\begin{abstract}
We study the total weight of weighted matchings in segment graphs, which is related to a question concerning generalized Chebyshev polynomials introduced by Vauchassade de Chaumont and Viennot and, more recently, investigated by Kim and Zeng. We prove that weighted matchings with sufficiently large node-weight cannot have equal total weight.
\end{abstract}

\section{Introduction}

Let $\operatorname{Seg}_{n}=(V, E)$ be the graph (i.e., the segment graph) with vertex set $V=[n]$ and $E$ the set of undirected edges $\{i, i+1\}$ with $1 \leq i \leq n-1$. A matching $\mu$ of $\operatorname{Seg}_{n}$ is a subset of edges with no two edges being connected by a common vertex. A node in $\mathrm{Seg}_{n}$ is called isolated (with respect to a given matching) if it is not contained in the matching. Given a matching, we put the weight $x$ on each isolated vertex and the weight -1 or $-a$ on each edge $\{i, i+1\}$, depending on whether $i$ is odd or even. Denote by $\mathcal{M}\left(\operatorname{Seg}_{n}\right)$ the set of all matchings of $\operatorname{Seg}_{n}$. The weighted matching polynomial is then given by

$$
U_{n}(x, a)=\sum_{\mu \in \mathcal{M}\left(\operatorname{Seg}_{n}\right)}(-1)^{|\mu|} a^{\operatorname{EIND}(\mu)} x^{n-2|\mu|},
$$




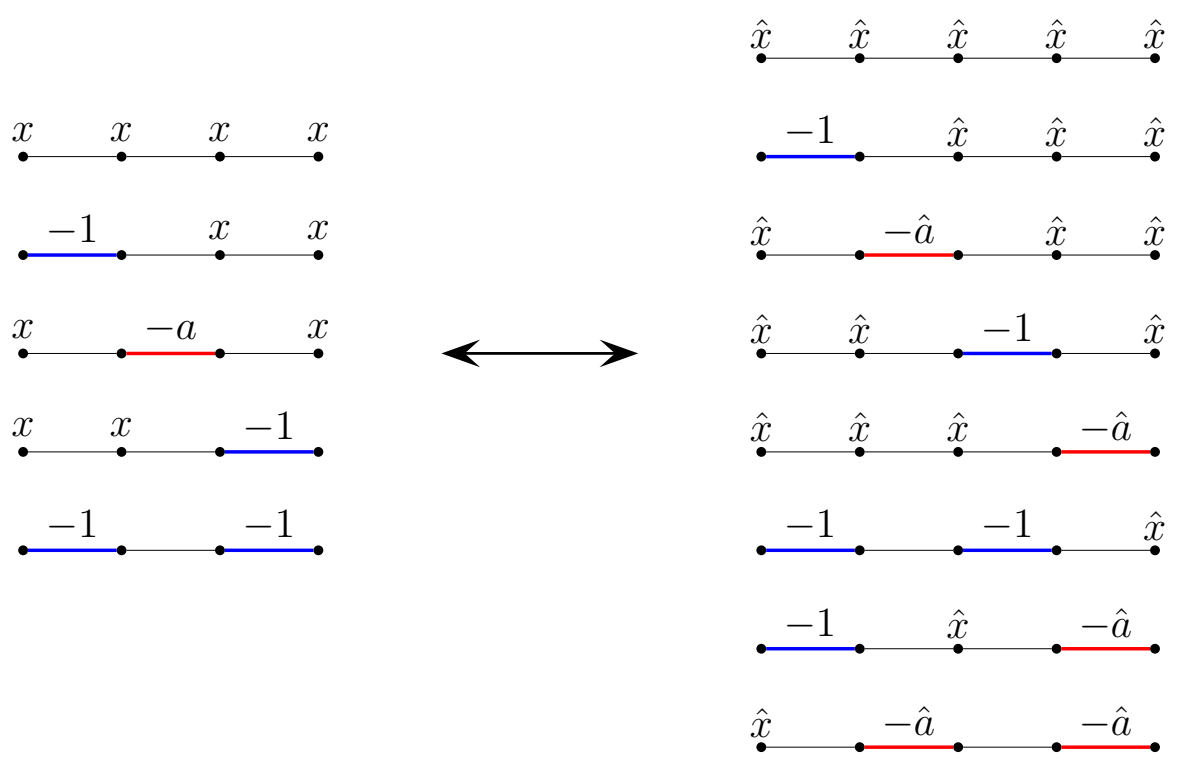

Figure 1: Weighted matchings of $\mathrm{Seg}_{4}$ and $\mathrm{Seg}_{5}$.

where $|\mu|$ denotes the number of edges of $\mu$ and $\operatorname{EIND}(\mu)$ the number of edges $\{i, i+1\}$ with $i$ even. The polynomials $U_{n}(x, a)$ came first up in some enumeration problems in molecular biology [15] and are generalized Chebyshev polynomials of the second kind because we get the classical (monic) Chebyshev polynomials of the second kind [14, p. 29] for $a=1$. Recently, Kim and Zeng [7] used (1) and a combinatorial interpretation of the corresponding moments to evaluate the linearization coefficients of certain products involving $U_{n}(x, a)$, which again generalize and refine results by De Sainte-Catherine and Viennot [4].

The purpose of this paper consists in studying these matching polynomials yet from another point of view. We are interested in simultaneous weighted matchings on segment graphs of different size, and ask how often the cumulative weight of these matchings, i.e. the corresponding weighted matching polynomials, can be made equal.

To clarify the problem, we first may take a closer look at a specific example, namely, at the weighted matching polynomials of $\mathrm{Seg}_{4}$ and $\mathrm{Seg}_{5}$ (see Fig. 1):

We see that

$$
\begin{gathered}
U_{4}(x, a)=x^{4}+(-1) x^{2}+(-a) x^{2}+(-1) x^{2}+(-1)^{2} \\
=x^{4}-(a+2) x^{2}+1, \\
\begin{aligned}
U_{5}(\hat{x}, \hat{a})=\hat{x}^{5}+(-1) \hat{x}^{3}+ & (-\hat{a}) \hat{x}^{3}+(-1) \hat{x}^{3}+(-\hat{a}) \hat{x}^{3}+(-1)^{2} \hat{x}+(-1)(-\hat{a}) \hat{x}+(-\hat{a})^{2} \hat{x} \\
= & x^{5}-2(\hat{a}+1) \hat{x}^{3}+\left(\hat{a}^{2}+\hat{a}+1\right) \hat{x} .
\end{aligned}
\end{gathered}
$$

Given $m, n, a$ and $\hat{a}$, how often can we choose $x$ and $\hat{x}$, such that the cumulative weights equalize? In other words, regarding our example, how many integral solutions does $U_{4}(x, a)=U_{5}(\hat{x}, \hat{a})$ have? 
It is well-known $[7,15]$ that the family of polynomials $\left\{U_{n}(x, a)\right\}$ satisfies a three-term recurrence equation, i.e.,

$$
U_{0}(x, a)=1, \quad U_{1}(x, a)=x, \quad U_{n+1}(x, a)=x U_{n}(x, a)-\lambda_{n} U_{n-1}(x, a),
$$

where $\lambda_{2 k}=a, \lambda_{2 k+1}=1$. Kim and Zeng [7] used Viennot's theory for orthogonal polynomials $[16,17]$ to derive the combinatorial model (1) from (2). Recently, McSorley, Feinsilver and Schott [9] provided a general framework for generating all orthogonal polynomials via vertex-matching-partition functions of some suitably labeled paths. The main point of the present work is to exhibit a close connection between the enumeration of a graph-theoretic quantity (i.e., weighted matchings) and a number-theoretic finiteness result, which here relies on the fact that $\left\{U_{n}(x, a)\right\}$ "almost" denotes a classical orthogonal polynomial family. Similar diophantine problems evolve from the enumeration of colored permutations [8] and from lattice point enumeration in polyhedra [1] (see [12] for a list of references). One of the first works studying a diophantine equation which arises from a combinatorial problem is due to Hajdu [6]; many papers on similar topics have appeared. In the present graph-theoretic context, however, it is crucial that $\left\{U_{n}(x, a)\right\}$ can be related to classical orthogonal polynomials. Note that by Favard's theorem [3], the polynomials $U_{n}(x, a)$ given in $(2)$ are orthogonal with respect to a positive-definite moment functional if and only if $a>0$.

Theorem 1.1. Let $a, \hat{a} \in \mathbb{Q}^{+}$and $m>n \geq 3$. Then the equation

$$
U_{m}(x, a)=U_{n}(\hat{x}, \hat{a})
$$

has only finitely many integral solutions $x, \hat{x}$ with the exception of the case

$$
m=6, \quad n=3, \quad a=9 / 2, \quad \hat{a}=59 / 4,
$$

where $x=t, \hat{x}=t^{2}-4$ with $t \in \mathbb{Z}$ is an infinite family of solutions. In other words, besides (3), matchings of segment graphs with sufficiently large node-weights cannot have equal total matching weight.

The paper is organized as follows. We first establish a differential equation of second order for $U_{n}(x, a)$ (Section 2), which may be of independent interest. We then apply an algorithm due to the first author [13] to characterize all polynomial decompositions $U_{n}(x, a)=r(q(x))$ with polynomials $r, q \in \mathbb{R}[x]$ and use a theorem due to Bilu and Tichy [2] to conclude (Section 3).

\section{Differential equation}

Recall that the Chebyshev polynomials of the second kind $U_{n}(x):=U_{n}(x, 1)$ are defined by

$$
U_{0}(x)=1, \quad U_{1}(x)=2 x, \quad U_{n+1}(x)=2 x U_{n}(x)-U_{n-1}(x) .
$$


Let $V_{n}(x)=U_{n}(x / 2)$ be the monic Chebyshev polynomials of the second kind. In what follows, we assume that $a \in \mathbb{R}^{+}$. From (2) we observe that there are polynomials $P_{n}(x, a) \in$ $\mathbb{R}[x]$ and $Q_{n}(x, a) \in \mathbb{R}[x]$ such that $U_{2 n+1}(x, a)=x P_{n}\left(x^{2}, a\right)$ and $U_{2 n}(x, a)=Q_{n}\left(x^{2}, a\right)$. Since

$$
U_{n+1}(x, a)=\left(x^{2}-a-1\right) U_{n-1}(x, a)-a U_{n-3}(x, a), \quad n \geq 3,
$$

by scaling with

$$
\begin{aligned}
W_{n}(x, a) & =P_{n}(\sqrt{a} x+a+1, a) /(\sqrt{a})^{n}, \\
S_{n}(x, a) & =Q_{n}(\sqrt{a} x+a+1, a) /(\sqrt{a})^{n},
\end{aligned}
$$

we derive from $(5)$ that $W_{n}(x, a)$ and $S_{n}(x, a)$ satisfy the recurrences:

$$
\begin{aligned}
& W_{0}(x, a)=1, \quad W_{1}(x, a)=x, \quad W_{n+1}(x, a)=x W_{n}(x, a)-W_{n-1}(x, a), \\
& S_{0}(x, a)=1, \quad S_{1}(x, a)=x+\sqrt{a}, \quad S_{n+1}(x, a)=x S_{n}(x, a)-S_{n-1}(x, a) .
\end{aligned}
$$

Thus, the polynomials $W_{n}(x, a)$ are independent of $a$ and equal the monic Chebyshev polynomials of the second kind $V_{n}(x)$, while the polynomials $S_{n}(x, a)$ are co-recursive versions of the polynomials $V_{n}(x)$ (see [5, sec. 2.1.2]). In general, co-recursive orthogonal polynomials can be easily rewritten in terms of the original non-shifted and the first associated polynomials (see [5, eq. (20)]). By straightforward calculations we therefore get the following representation for $U_{n}(x, a)$.

Proposition 2.1. We have

$$
U_{n}(x, a)= \begin{cases}(\sqrt{a})^{k} x V_{k}\left(\frac{x^{2}-a-1}{\sqrt{a}}\right), & n=2 k+1 ; \\ (\sqrt{a})^{k}\left\{V_{k}\left(\frac{x^{2}-a-1}{\sqrt{a}}\right)+\sqrt{a} V_{k-1}\left(\frac{x^{2}-a-1}{\sqrt{a}}\right)\right\}, & n=2 k .\end{cases}
$$

Taking into account the explicit coefficient formula for Chebyshev polynomials of the second kind (see e.g. [10]) we obtain

$$
U_{n}(x, a)=x^{n}+\varepsilon_{2}^{(n)} x^{n-2}+\varepsilon_{4}^{(n)} x^{n-4}+\cdots,
$$

where

$$
\begin{aligned}
& \varepsilon_{2}^{(n)}= \begin{cases}-\frac{1}{2}(a+1) n+a, & n \text { even; } \\
-\frac{1}{2}(a+1)(n-1), & n \text { odd },\end{cases} \\
& \varepsilon_{4}^{(n)}= \begin{cases}\frac{1}{8}(n-2)\left(n(a+1)^{2}-4 a^{2}-8 a\right), & n \text { even; } \\
\frac{1}{8}(n-3)\left(n(a+1)^{2}-a^{2}-6 a-1\right), & n \text { odd } .\end{cases}
\end{aligned}
$$

Similar expressions can be also given for $\varepsilon_{6}^{(n)}, \varepsilon_{8}^{(n)}$ and $\varepsilon_{10}^{(n)}$ (see Appendix). It is well-known [14] that Chebyshev polynomials of the second kind satisfy a second order differential equation with polynomial coefficients of degree $\leq 2$. With the aid of Proposition 2.1, it is a direct calculation to come up with a differential equation also for $U_{n}(x, a)$ (however, with polynomial coefficients of higher order). 
Proposition 2.2. The polynomials $U_{n}(x, a)$ satisfy

$$
A(x) U_{n}(x, a)+B(x) U_{n}^{\prime}(x, a)+C(x) U_{n}^{\prime \prime}(x, a)=0,
$$

where

- $n$ even:

$$
\begin{aligned}
& A(x)=-n(n+1)(n+2) x^{5}+3 n(n+2)(a-1) x^{3}, \\
& B(x)=3(n+1) x^{6}-5(a-1) x^{4}-(a-1)\{(3 n-1) a-(3 n+7)\} x^{2}+(a-1)^{3} \text {, } \\
& C(x)=(n+1) x^{7}-\{(2 n+3) a+(2 n+1)\} x^{5} \\
& +(a-1)\{(n+3) a-(n-1)\} x^{3}-(a-1)^{3} x ;
\end{aligned}
$$

- $n$ odd:

$$
\begin{aligned}
& A(x)=-n(n+2) x^{4}+3(a-1)^{2}, \\
& B(x)=3 x^{5}-3(a-1)^{2} x, \\
& C(x)=x^{6}-2(a+1) x^{4}+(a-1)^{2} x^{2} .
\end{aligned}
$$

In the next lemma we make use of the differential equation given in Proposition 2.2.

Lemma 2.3. Let $a \in \mathbb{R}^{+}$and $U_{n}(x, a)=r(q(x))$ with $r, q \in \mathbb{R}[x]$ and $\min (\operatorname{deg} r, \operatorname{deg} q) \geq$ 2. Then $\operatorname{deg} q \leq 6$.

Proof. We use a powerful method due to Sonin, Pólya and Szegö; for more details see [12, 13]. Define the Sonin-type function

$$
h(x)=U_{n}(x, a)^{2}+\frac{C(x)}{A(x)} U_{n}^{\prime}(x, a)^{2},
$$

which by Proposition 2.2 satisfies $h^{\prime}(x)=-\frac{\omega(x)}{A(x)^{2}} U_{n}^{\prime}(x, a)^{2}$ with

$$
\omega(x)=\left(2 B(x)-C^{\prime}(x)\right) A(x)+C(x) A^{\prime}(x) .
$$

If $n$ is even then

$$
\begin{aligned}
\omega(x)= & x^{5}\left(-4 n(n+1)^{2}(n+2) x^{6}+16 n(n+1)(n+2)(a-1) x^{4}\right. \\
& \left.+4 n(n+2)\left(n^{2}+2 n-5\right)(a-1)^{2} x^{2}-8 n(n+2)(a-1)^{2}(2 a n-a-2 n-5)\right) .
\end{aligned}
$$

By Descartes' rule of signs [10, p. 7] and $a \in \mathbb{R}^{+}$this polynomial has at most five distinct real zeroes, thus $h^{\prime}(x)$ changes sign at most five times. Since $U_{n}(x, a)$ has only simple real zeroes for $a>0$, by Rolle's theorem so does $U_{n}^{\prime}(x, a)$. We therefore have $\operatorname{deg} \operatorname{gcd}\left(U_{n}(x, a)-\right.$ $\left.\zeta, U_{n}^{\prime}(x, a)\right) \leq 6$, for all $\zeta \in \mathbb{C}$. Now, suppose a non-trivial decomposition $U_{n}(x, a)=$ $r(q(x))$ and denote by $\zeta_{0}$ a zero of $r^{\prime}$, which exists by $\operatorname{deg} r \geq 2$. Then both $U_{n}(x, a)-r\left(\zeta_{0}\right)$ and $U_{n}^{\prime}(x, a)$ are divisible by $q(x)-r\left(\zeta_{0}\right)$. Thus,

$$
\operatorname{deg} q \leq \operatorname{deg}(q-\zeta) \leq \operatorname{deg} \operatorname{gcd}\left(U_{n}(x, a)-\zeta, U_{n}^{\prime}(x, a)\right) \leq 6,
$$

which completes the proof for $n$ even. If $n$ is odd then

$$
\omega(x)=x\left(-4 n(n+2) x^{8}+4(a-1)^{2} n(n+2) x^{4}+24(a+1)(a-1)^{2} x^{2}-24(a-1)^{4}\right),
$$

and a similar argument yields the result. 


\section{Polynomial decomposition}

This section is devoted to a complete characterization of polynomial decompositions of $U_{n}(x, a)$. By a polynomial decomposition of $p(x) \in \mathbb{R}[x]$ we mean $p(x)=r \circ q(x)$ with $r, q \in \mathbb{R}[x]$ and $\min (\operatorname{deg} r, \operatorname{deg} q) \geq 2$. We call two decompositions $p=r_{1} \circ q_{1}=r_{2} \circ q_{2}$ equivalent, if there is a linear polynomial $\kappa$ such that $r_{2}=r_{1} \circ \kappa$ and $q_{2}=\kappa^{-1} \circ q_{1}$. A polynomial $p$ is said to be indecomposable, if there is no polynomial decomposition of $p$.

Lemma 3.1. The generalized Chebyshev polynomials $U_{n}(x, a)$ are indecomposable (up to equivalence) except in the following cases:

(i) $n=2 k, k \geq 2$; then $U_{n}(x, a)=r\left(x^{2}\right)$ and $r(x)$ is indecomposable unless $(i i i)$.

(ii) $n=6, a=\frac{3}{4}$; then $U_{6}\left(x, \frac{3}{4}\right)=\left(x^{2}-1\right) \circ\left(x^{3}-\frac{9}{4} x\right)$.

(iii) $n=8, a=4$; then $U_{8}(x, 4)=\left(x^{2}+14 x+1\right) \circ\left(x^{4}-8 x^{2}\right)$.

In view of Lemma 2.3, we have to show that $U_{n}(x, a)=r(q(x))$ with $3 \leq \operatorname{deg} q \leq 6$ leads - in general - to a contradiction. A well-arranged way to equate the (parametric) coefficients on both sides of the decomposition equation is via the algorithmic approach (using Gröbner techniques) presented in [13, sec. 4]. We shortly recall and outline the procedure for $\operatorname{deg} q=3$ and $n$ even, where we find (ii) in Lemma 3.1. The other cases are similar (for instance, for $\operatorname{deg} q=4$ we consider $\left[x^{4 k-6}\right]=\left[x^{4 k-10}\right]=0$ etc.).

By [12, Proposition 3.3] we can calculate a polynomial $\hat{q}(x)$ of degree three from the data given in (6) which has the following property: If $U_{n}(x, a)=r(q(x))$ with $\operatorname{deg} q=3$ and $q(0)=0$, lcoeff $(q(x))=1$ then necessarily $q(x) \equiv \hat{q}(x)$. In other words, $\hat{q}(x)$ is the only (normed) candidate of degree three. According to [13, Algorithm 1] we here get

$$
\hat{q}(x)=x^{3}-\frac{3 k(a+1)-2 a}{2 k} x,
$$

such that $U_{3 k}(x, a)=\hat{q}(x)^{k}+\mathcal{R}(x)$ with $\mathcal{R}(x)=\beta_{1} x^{3 k-4}+$ terms of lower order. If there is a decomposition with a right component of degree three, then necessarily $\beta_{1}=0$, which gives the equation

$$
3 k^{2} a^{2}-6 k^{2} a+3 k^{2}-8 a^{2} k+4 a k+4 a^{2}=0 .
$$

Therefore, assuming $k>2$, we may suppose that

$$
U_{3 k}(x, a)=\hat{q}(x)^{k}+\beta_{2} \hat{q}(x)^{k-2}+\mathcal{R}_{1}(x),
$$

where $\operatorname{deg} \mathcal{R}_{1} \leq 3 k-8$. Indeed, the coefficient $\left[x^{3 k-8}\right]$ in $\mathcal{R}_{1}(x)$ must be zero. This yields

$$
\begin{gathered}
(k-2)\left(162 k^{5} a^{4}+162 k^{5}-324 k^{5} a^{2}+378 k^{4} a^{2}-189 k^{4}-324 k^{4} a^{3}+108 k^{4} a\right. \\
-837 k^{4} a^{4}-72 a k^{3}+72 a^{2} k^{3}+648 a^{3} k^{3}+1656 a^{4} k^{3}+72 a^{2} k^{2} \\
\left.-624 a^{3} k^{2}-1512 a^{4} k^{2}+480 a^{3} k+608 a^{4} k-80 a^{4}\right)=0 .
\end{gathered}
$$

Obviously, $k=2, a=3 / 4$ is a solution of (8). On the other hand, it is easy to see that the solutions of (8) and (9) for $k \geq 3$ are not admissible (this can be checked, for instance, with the help of the Groebner package and the Solve command in MAPLE). 
Corollary 3.2. Let $a, \hat{a} \in \mathbb{R}^{+}$and $m>n \geq 3$. Then there does not exist a polynomial $P(x) \in \mathbb{R}[x]$ such that

$$
U_{m}(x, a)=U_{n}(P(x), \hat{a})
$$

with the exception of the case

$$
m=6, \quad n=3, \quad a=\frac{9}{2}, \quad \hat{a}=\frac{59}{4}, \quad P(x)=x^{2}-4 .
$$

Proof. By Lemma 3.1 every decomposition of $U_{m}(x, a)=r(q(x))$ with $\operatorname{deg} q \geq 3$ implies $\operatorname{deg} r \leq 2$, which is not allowed by $n \geq 3$. Therefore, we may assume that $P(x)=\alpha x^{2}+\beta$ for some $\alpha, \beta \in \mathbb{R}$. First, suppose $n \geq 5$. Equating $\left[x^{m-2}\right],\left[x^{m-4}\right],\left[x^{m-6}\right],\left[x^{m-8}\right]$ and $\left[x^{m-10}\right]$ on both sides of (10) yields a contradiction (see the Appendix for the corresponding quantities). It is straightforward to exclude also the case $n=4$. Finally, for $n=3$ we have $\alpha=1$ and the coefficient equations

$$
-3-2 a=3 \beta, \quad a^{2}+2 a+3=3 \beta^{2}-\hat{a}-1, \quad-1=\beta^{3}-(1+\hat{a}) \beta,
$$

which yield $(\beta, a, \hat{a})=(-1,0,-1)$ or $(\beta, a, \hat{a})=(-4,9 / 2,59 / 4)$. Only the latter solution is admissible.

As for the final step, we recall the finiteness theorem due to Bilu and Tichy [2]. Again, for more details we refer to [12]. First some more notation is needed. Let $\gamma, \delta \in \mathbb{Q} \backslash\{0\}$, $r, q, s, t \in \mathbb{Z}^{+} \cup\{0\}$ and $v(x) \in \mathbb{Q}[x]$. Denote by $D_{s}(x, a)$ the Dickson polynomial of the first kind of degree $s$ defined by

$$
\begin{aligned}
D_{0}(x, a) & =2, \quad D_{1}(x, a)=x \\
D_{n+1}(x, a) & =x D_{n}(x, a)-a D_{n-1}(x, a), \quad n \geq 1,
\end{aligned}
$$

which satisfies $D_{n}(x, a)=x^{n}+d_{2}^{(n)} x^{n-2}+d_{4}^{(n)} x^{n-4}+\cdots$, where

$$
d_{2 k}^{(n)}=\frac{n}{n-k}\left(\begin{array}{c}
n-k \\
k
\end{array}\right)(-a)^{k} .
$$

To state the result, we also need the notion of five types of so-called standard pairs, which are pairs of polynomials of some special shape. To begin with, a standard pair of the first kind is of the type $\left(x^{q}, \gamma x^{r} v(x)^{q}\right)$ (or switched), where $0 \leq r<q, \operatorname{gcd}(r, q)=1$ and $r+\operatorname{deg} v>0$. A standard pair of the second kind is given by $\left(x^{2},\left(\gamma x^{2}+\delta\right) v(x)^{2}\right)$ (or switched). A standard pair of the third kind is $\left(D_{s}\left(x, \gamma^{t}\right), D_{t}\left(x, \gamma^{s}\right)\right)$ with $s, t \geq 1$ and $\operatorname{gcd}(s, t)=1$. A standard pair of the fourth kind is $\left(\gamma^{-s / 2} D_{s}(x, \gamma),-\delta^{-t / 2} D_{t}(x, \delta)\right.$ ) (or switched) with $s, t \geq 1$ and $\operatorname{gcd}(s, t)=2$. Finally, a standard pair of the fifth kind is of the form $\left(\left(\gamma x^{2}-1\right)^{3}, 3 x^{4}-4 x^{3}\right)$ (or switched).

The following (less strong) version of the theorem of Bilu and Tichy [2] is sufficient for our purposes: 
Theorem 3.3 (Bilu/Tichy [2]). Let $f(x), g(x) \in \mathbb{Q}[x]$ be non-constant polynomials and assume that there do not exist linear polynomials $\kappa_{1}, \kappa_{2} \in \mathbb{Q}[x]$, a polynomial $\varphi(x) \in \mathbb{Q}[x]$ and a standard pair $\left(\mathfrak{f}_{1}, \mathfrak{g}_{1}\right)$ such that

$$
f=\varphi \circ \mathfrak{f}_{1} \circ \kappa_{1} \quad \text { and } \quad g=\varphi \circ \mathfrak{g}_{1} \circ \kappa_{2},
$$

then the equation $f(x)=g(y)$ has only finitely many integral solutions.

We stress the fact that the proof of Theorem 3.3 is based - beside other tools - on Siegel's theorem on integral points on algebraic curves [11] and is therefore ineffective. This means that we have no upper bound available for the size of solutions $x, y$. The standard pairs make up the exceptional cases where one can find an infinite parametric solution set. According to Theorem 3.3 we here have to check the decompositions of shape (13) whether they match with those given in Lemma 3.1.

To start with, consider the standard pair of the first kind and $U_{m}(\alpha x+\beta, a)=\varphi\left(x^{q}\right)$. If $q \geq 3$ then $\beta=0$ and $\varepsilon_{2}^{(m)} \alpha^{m-2}=0$, a contradiction. If $q=2$ then since $m, n \geq 3$ we have $\operatorname{deg} \varphi \geq 2$. We distinguish two cases. If $\operatorname{deg} \varphi=2$ then we are led to the system of equations

$$
\begin{aligned}
& U_{4}\left(\alpha_{1} x+\beta_{1}, a\right)=e_{2} x^{4}+e_{1} x^{2}+e_{0}, \\
& U_{6}\left(\alpha_{2} x+\beta_{2}, \hat{a}\right)=e_{2} x^{2}\left(v_{1} x+v_{0}\right)^{4}+e_{1} x\left(v_{1} x+v_{0}\right)^{2}+e_{0},
\end{aligned}
$$

or, respectively, with switched parameters $a, \hat{a}$. Equating coefficients on both sides gives a contradiction. ${ }^{1}$

If $\operatorname{deg} \varphi \geq 3$ then $\operatorname{deg}\left(x^{r} v(x)^{q}\right)=1$ which by Corollary 3.2 gives $m=6, n=3, a=9 / 2$ and $\hat{a}=59 / 4$. A similar conclusion holds for $q=1$.

A standard pair of the second kind is not possible as well. Since $m \neq n$, we have $\operatorname{deg} v(x) \geq 1$. Again, we distinguish two cases. If $\operatorname{deg} v(x) \geq 2$ then $\varphi(x)$ is linear, a contradiction to $m, n \geq 3$. On the other hand, if $\operatorname{deg} v(x)=1$ we have the two equations (resp. with switched parameters),

$$
\begin{aligned}
& U_{4}\left(\alpha_{1} x+\beta_{1}, a\right)=e_{2} x^{4}+e_{1} x^{2}+e_{0}, \\
& U_{8}\left(\alpha_{2} x+\beta_{2}, \hat{a}\right)=e_{2}\left(\gamma x^{2}+\delta\right)^{2}\left(v_{1} x+v_{0}\right)^{4}+e_{1}\left(\gamma x^{2}+\delta\right)\left(v_{1} x+v_{0}\right)^{2}+e_{0} .
\end{aligned}
$$

Again a contradiction arises.

Next, consider the standard pair of the fifth kind and suppose $U_{m}(\alpha x+\beta, a)=\varphi\left(\left(\gamma x^{2}-\right.\right.$ $\left.1)^{3}\right)$. This implies that $\varphi(x)$ is linear. Again, a contradiction arises, since $U_{m}^{\prime}(x, a)$ only has simple roots whereas the derivative of the right-hand-side polynomial has a triple root.

It remains to treat the standard pairs of the third and fourth kind. Suppose a standard pair of the third kind, namely $U_{m}\left(\alpha_{1} x+\beta_{1}\right)=\varphi\left(D_{s}\left(x, \gamma^{t}\right)\right)$ and $U_{n}\left(\alpha_{2} x+\beta_{2}\right)=$ $\varphi\left(D_{t}\left(x, \gamma^{s}\right)\right)$. By $\operatorname{gcd}(s, t)=1$ and Lemma 3.1 we see that $\operatorname{deg} \varphi \leq 2$ (leaving aside the

\footnotetext{
${ }^{1}$ Again, we used safe Groebner computations with MAPLE to conclude for (14) and (15).
} 
case (11)). First, let $\varphi(x)$ be linear. Assume $m \geq 7$ and $U_{m}\left(\alpha_{1} x+\beta_{1}\right)=e_{1} D_{s}(x, \delta)+e_{0}$ with $\delta=\gamma^{t}$. Then using (12) the coefficient equations

$$
\varepsilon_{2 k}^{(m)}=\alpha_{1}^{2 k} d_{2 k}^{(m)}, \quad k=1,2,3
$$

yield a contradiction. Therefore,

$$
(m, n) \in\{(6,5),(5,4),(5,3),(4,3)\} .
$$

Suppose $m=5$. Then (16) with $k=1,2$ yields $a=(3 \pm \sqrt{5}) / 2 \notin \mathbb{Q}$, a contradiction. Let $U_{4}\left(\alpha_{1} x+\beta_{1}, a\right)=e_{1}\left(x^{4}-4 \gamma^{3} x^{2}+2 \gamma^{6}\right)+e_{0}$ and $U_{3}\left(\alpha_{2} x+\beta_{2}, \hat{a}\right)=e_{1}\left(x^{3}-3 \gamma^{4} x\right)+e_{0}$. This gives $e_{0}=0, e_{1}=\alpha_{1}^{4}$ and the contradiction $2 \alpha_{1}^{4} \gamma^{6}=1$. Now, suppose $\varphi(x)=$ $e_{2} x^{2}+e_{1} x+e_{0}$. By $(s, t)=1$ and Lemma 3.1 we have an equation similar to

$$
U_{6}\left(\alpha_{1} x+\beta_{1}\right)=e_{2} D_{3}(x, \delta)^{2}+e_{1} D_{3}(x, \delta)+e_{0},
$$

which directly leads to a contradiction.

Finally, suppose a standard pair of the fourth kind. Again, we conclude $\operatorname{deg} \varphi \leq 2$. First, let $\operatorname{deg} \varphi=1$. From the discussion above we see that $(m, n)=(6,4)$. Thus,

$$
\begin{aligned}
& U_{6}\left(\alpha_{1} x+\beta_{1}, a\right)=e_{1}\left(\frac{x^{6}}{\gamma_{1}^{3}}-\frac{6 x^{4}}{\gamma_{1}^{2}}+\frac{9 x^{2}}{\gamma_{1}}-2\right)+e_{0}, \\
& U_{4}\left(\alpha_{2} x+\beta_{2}, \hat{a}\right)=e_{1}\left(-\frac{x^{4}}{\gamma_{2}^{2}}+\frac{4 x^{2}}{\gamma_{2}}-2\right)+e_{0} .
\end{aligned}
$$

This gives $\alpha_{2}^{4} \gamma_{2}^{2}=-e_{1}$ and $-(2 a+3) \alpha_{1}^{4} \gamma_{1}^{2}=-6 e_{1}$ which implies $a<0$, a contradiction. A similar argument also applies for $\operatorname{deg} \varphi=2$. This completes the proof of Theorem 1.1.

Remark. It causes no great difficulty to replace the edge weight -1 by $-\beta$ with $\beta \in \mathbb{Q}^{+}$ in (1) and to conclude in a similar way. Both Proposition 2.2 and Lemma 3.1 can be appropriately generalized. As the focus of the paper is on the cross connection between Diophantine properties and graph quantities, we here omit the details for the general case.

\section{Appendix}

We here append some more upper coefficients of $U_{n}(x, a)$ which are needed in the proof of Corollary 3.2 and in the last section.

$$
\varepsilon_{6}^{(n)}=\left\{\begin{array}{cc}
-\frac{1}{48}(n-4)\left(3 n^{2} a+n^{2}+n^{2} a^{3}+3 n^{2} a^{2}-24 n a-2 n-30 n a^{2}\right. & \\
\left.-8 n a^{3}+72 a^{2}+36 a+12 a^{3}\right), & n \text { even } \\
-\frac{1}{48}(n-3)(n-5)(a+1)\left(n a^{2}-a^{2}-14 a+2 n a+n-1\right), & n \text { odd }
\end{array}\right.
$$




$$
\begin{gathered}
\varepsilon_{8}^{(n)}=\left\{\begin{array}{cc}
\frac{1}{384}(n-4)(n-6)\left(n^{2} a^{4}+4 n^{2} a^{3}+6 n^{2} a^{2}+4 n^{2} a+n^{2}-40 n a-10 n a^{4}\right. \\
\left.-84 n a^{2}-2 n-56 n a^{3}+64 a+16 a^{4}+288 a^{2}+192 a^{3}\right), & n \text { even; } \\
\frac{1}{384}(n-5)(n-7)\left(n^{2} a^{4}+4 n^{2} a^{3}+6 n^{2} a^{2}+4 n^{2} a+n^{2}-4 n a^{4}-72 n a^{2}\right. & \\
\left.-40 n a-4 n-40 n a^{3}+84 a+210 a^{2}+3 a^{4}+3+84 a^{3}\right), & n \text { odd. }
\end{array}\right. \\
\varepsilon_{10}^{(n)}=\left\{\begin{array}{cc}
-\frac{1}{3840}(n-6)(n-8)\left(10 n^{3} a^{3}+n^{3} a^{5}+5 n^{3} a^{4}+n^{3}+10 n^{3} a^{2}+5 n^{3} a-80 n^{2} a\right. \\
-110 n^{2} a^{4}-6 n^{2}-220 n^{2} a^{2}-16 n^{2} a^{5}-240 n^{2} a^{3}+340 n a \\
+1520 n a^{2}+68 n a^{5}+760 n a^{4}+8 n+1880 n a^{3}-3200 a^{2} & n \text { even;} \\
\left.-400 a-1600 a^{4}-4800 a^{3}-80 a^{5}\right), & n \text { odd. } \\
-\frac{1}{3840}(n-5)(n-7)(n-9)(a+1)\left(n^{2} a^{4}-4 n a^{4}+3 a^{4}-56 n a^{3}+4 n^{2} a^{3}\right. & \\
+132 a^{3}+6 n^{2} a^{2}-104 n a^{2}+498 a^{2}-56 n a+4 n^{2} a+132 a & \\
\left.+n^{2}-4 n+3\right), &
\end{array}\right.
\end{gathered}
$$

\section{Acknowledgement}

The first author is a recipient of an APART-fellowship of the Austrian Academy of Sciences at the University of Waterloo, Canada. Support has also been granted by the Austrian Science Foundation (FWF), project S9604, "Analytic and Probabilistic Methods in Combinatorics". The second author is supported by la Région Rhône-Alpes through the program "MIRA Recherche 2008", project 0803414701.

\section{References}

[1] Y. Bilu, T. Stoll, R.F. Tichy, Octahedrons with equally many lattice points, Period. Math. Hungar. 40 (2000), no. 2, 229-238.

[2] Y. Bilu, R.F. Tichy, The Diophantine equation $f(x)=g(y)$, Acta Arith. 95 (2000), 261-288.

[3] T.S. Chihara, An Introduction to Orthogonal Polynomials, Gordon and Breach, New York, 1978.

[4] M. De Sainte-Catherine, X.G. Viennot, Combinatorial Interpretation of Integrals of Products of Hermite, Laguerre and Tchebycheff Polynomials, Lecture Notes in Mathematics, vol. 1171, Springer-Verlag, 1985, pp. 120-128.

[5] M. Foupouagnigni, W. Koepf, A. Ronveaux, Factorization of forth-order differential equations for perturbed classical orthogonal polynomials, J. Comput. Appl. Math. 162 (2004), 299-326.

[6] L. Hajdu, On a Diophantine equation concerning the number of integer points in special domains, Acta Math. Hungar. 78 (1998), 59-70.

[7] D. Kim, J. Zeng, Combinatorics of generalized Tchebycheff polynomials, European J. Combin. 24 (2003), 499-509.

[8] P. Kirschenhofer, O. Pfeiffer, A class of combinatorial Diophantine equations, Sém. Lothar. Combin. 44 (2000), Art. B44h, 7 pp. (electronic). 
[9] J.P. McSorley, P. Feinsilver, R. Schott, Generating orthogonal polynomials and their derivatives using vertex|matching-partitions of graphs, Ars Combin. 87 (2008), 75-95.

[10] Ze V.V. Prasolov, Polynomials, translated from the 2001 Russian second edition by Dimitry Leites. Algorithms and Computation in Mathematics, Springer, Berlin, 2004.

[11] C.L. Siegel, Über einige Anwendungen Diophantischer Approximationen, Abh. Preuss. Akad. Wiss. Phys.-Math. Kl. (1929), no. 1, 209-266.

[12] T. Stoll, Complete decomposition of Dickson-type recursive polynomials and related Diophantine equations, J. Number Theory, 128 (2008), 1157-1181.

[13] T. Stoll, Decomposition of perturbed Chebyshev polynomials, J. Comp. Appl. Math. 214 (2008), 356-370.

[14] G. Szegő, Orthogonal polynomials, American Mathematical Society Colloquium Publications, vol. 23, Fourth edition, Providence, R.I., 1975.

[15] M. Vauchassade de Chaumont, X.G. Viennot, Polynômes orthogonaux et problèmes d'énumération en biologie moléculaire, Sém. Lothar. Combin. B081(1984) 8.

[16] X.G. Viennot, Une théorie combinatoire des polynômes orthogonaux généraux, Notes de conférences, UQAM, Montréal, 1984.

[17] X.G. Viennot, A combinatorial theory for general orthogonal polynomials with extensions and applications, Orthogonal polynomials and applications, Lecture Note in Math., 1171, Springer, Berlin, 1985, pp. 139-157. 